\title{
Characterization of the gut microbiota of Kawasaki disease patients by metagenomic analysis
}

\author{
Akiko Kinumaki ${ }^{1,2}$, Tsuyoshi Sekizuka ${ }^{2}$, Hiromichi Hamada ${ }^{3}$, Kengo Kato ${ }^{2}$, \\ Akifumi Yamashita ${ }^{2}$ and Makoto Kuroda ${ }^{2 *}$ \\ ${ }^{1}$ Department of Pediatrics, Graduate School of Medicine, University of Tokyo, Bunkyo-ku, Japan, ${ }^{2}$ Laboratory of Bacterial \\ Genomics, Pathogen Genomics Center, National Institute of Infectious Diseases, Shinjuku-ku, Japan, ${ }^{3}$ Department of \\ Pediatrics, Faculty of Medicine, Yachiyo Medical Center, Tokyo Women's Medical University, Yachiyo, Japan
}

OPEN ACCESS

Edited by:

Roy D. Sleator,

Cork Institute of Technology, Ireland

Reviewed by:

Suleyman Yildirim

Istanbul Medipol University

International School

of Medicine, Turkey

Michael S. Allen,

University of North Texas Health

Science Center, USA

*Correspondence:

Makoto Kuroda,

Pathogen Genomics Center, National Institute of Infectious Diseases, 1-23-1

Toyama, Shinjuku-ku,

Tokyo 162-8640, Japan

makokuro@nih.go.jp

Specialty section: This article was submitted to

Evolutionary and Genomic Microbiology,

a section of the journal

Frontiers in Microbiology

Received: 11 March 2015

Accepted: 27 July 2015

Published: 11 August 2015

Citation:

Kinumaki A, Sekizuka T, Hamada H, Kato K, Yamashita $A$ and Kuroda $M$

(2015) Characterization of the gut microbiota of Kawasaki disease

patients by metagenomic analysis.

Front. Microbiol. 6:824

doi: 10.3389/fmicb.2015.00824
Kawasaki disease (KD) is an acute febrile illness of early childhood. Previous reports have suggested that genetic disease susceptibility factors, together with a triggering infectious agent, could be involved in KD pathogenesis; however, the precise etiology of this disease remains unknown. Additionally, previous culture-based studies have suggested a possible role of intestinal microbiota in KD pathogenesis. In this study, we performed metagenomic analysis to comprehensively assess the longitudinal variation in the intestinal microbiota of $28 \mathrm{KD}$ patients. Several notable bacterial genera were commonly extracted during the acute phase, whereas a relative increase in the number of Ruminococcus bacteria was observed during the non-acute phase of KD. The metagenomic analysis results based on bacterial species classification suggested that the number of sequencing reads with similarity to five Streptococcus spp. (S. pneumonia, pseudopneumoniae, oralis, gordonii, and sanguinis), in addition to patient-derived Streptococcus isolates, markedly increased during the acute phase in most patients. Streptococci include a variety of pathogenic bacteria and probiotic bacteria that promote human health; therefore, this further species discrimination could comprehensively illuminate the KD-associated microbiota. The findings of this study suggest that KD-related Streptococci might be involved in the pathogenesis of this disease.

Keywords: Kawasaki disease, gut microbiota, metagenomic analysis, Streptococcus, mitis group

\section{Introduction}

Kawasaki disease (KD) is an acute febrile illness of early childhood. The principal pathology is systemic vasculitis with coronary artery involvement, and $\mathrm{KD}$ is the leading cause of acquired heart disease in developed countries. It was originally described by Dr. Tomisaku Kawasaki in 1967 (Kawasaki, 1967), and it is known to occur worldwide in children of all races. However, as the etiology of KD remains unknown, no specific biological markers for diagnostic testing

Abbreviations: BLAST, Basic Local Alignment Search Tool; BWA-SW, Burrows-Wheeler Aligner's Smith-Waterman Alignment; CFU, colony forming unit; EDTA, ethylenediaminetetraacetic acid; FASTQ, a text-based format for storing both a biological sequence (usually a nucleotide sequence) and its corresponding quality scores; KD, Kawasaki disease; LPS, lipopolysaccharide; LEfSe, linear discriminant analysis (LDA) coupled with effect size measurements; LDA, linear discriminant analysis; MEGAN, MEtaGenome Analyzer; PCA, Principal component analysis; PERMANOVA, Permutational Multivariate Analysis Of Variance; TCR, T-cell receptor. 
have been characterized to date. The diagnosis of KD is based on the following six clinical features: fever lasting for at least 5 days, changes in the extremities, polymorphous exanthem, bilateral conjunctival injection without exudate, changes in the lips and oral cavity, and cervical lymphadenopathy (Newburger et al., 2004). Although the simultaneous intravenous infusion of gamma globulin and aspirin is effective in reducing systemic inflammation and preventing coronary artery involvement, coronary abnormalities still develop in $\sim 5 \%$ of affected children, and some patients show no response to this therapy (Newburger et al., 1991).

The annual incidence of $\mathrm{KD}$ is increasing rapidly in Japan, with 239.6/100,000 children under the age of 5 years affected in 2010. This incidence is by far the highest rate worldwide (Nakamura et al., 2012), and the risk of KD in siblings of affected children is significantly higher than that in the general population (Fujita et al., 1989). The annual incidence rates are also relatively high in other East Asian countries (with $113.1 / 100,000$ children under the age of 5 years affected in Korea and 69/100,000 in Taiwan) but are low in Europe and North America (with 4.9-15.2/100,000 children under the age of 5 years affected in European countries and 19-26.2/100,000 in North American countries) (Uehara and Belay, 2012). A higher rate of KD has been reported in Hawaiian children of Japanese descent compared with those of European descent (Holman et al., 2010), suggesting the importance of genetic factors in disease susceptibility.

Epidemiological studies have shown that the age-specific incidence rate of $\mathrm{KD}$ is the highest among children aged 611 months and that $88.4 \%$ of $\mathrm{KD}$ patients are less than 1 year of age (Uehara and Belay, 2012). Interestingly, a seasonal variation in the number of affected $\mathrm{KD}$ patients has been observed (Nakamura et al., 2008). These findings suggest that an infectious agent may trigger this disease; however, its etiology remains unknown. A GWAS of KD in Japanese patients has revealed susceptibility loci related to immune disorders and a human leukocyte antigen; such extensive studies will facilitate characterization of the pathogenesis and pathophysiology (Onouchi, 2012; Onouchi et al., 2012).

Previous reports have suggested that an elevation in lipopolysaccharide (LPS, endotoxin)-binding neutrophils or plasma proteins (Takeshita et al., 1999, 2002b), antibody reactivity against mycobacterial heat-shock protein (HSP65) in convalescent sera (Yokota et al., 1993), and unique TCR $\mathrm{V} \beta$ expansion by certain superantigens (SAg) in KD patients (Abe et al., 1992; Yoshioka et al., 1999) might be involved in $\mathrm{KD}$ pathogenesis. Case reports have suggested that these factors are attributed to the presence of secondary infections with various pathogens, including Streptococcus pyogenes, Staphylococcus aureus, Mycoplasma pneumoniae, Chlamydia pneumoniae, Klebsiella pneumoniae, adenovirus, EpsteinBarr virus, parvovirus B19, herpesvirus 6, parainfluenza virus, measles, rotavirus, dengue virus, varicella zoster virus, cytomegalovirus, and influenza virus (Johnson and Azimi, 1985; Catalano-Pons et al., 2005; Wang et al., 2005; Joshi et al., 2011; Principi et al., 2013). In animal models, exposure to the Lactobacillus bacterial cell wall (Duong et al., 2003), immunization with bacillus Calmette-Guérin (BCG) (Nakamura et al., 2007), or exposure to the Candida albicans water-soluble fraction (Nagi-Miura et al., 2004; Ohno, 2004) has been shown to induce vasculitis and coronary arteritis. These observations further suggest that infectious agents promote the onset of KD.

The intestinal microbiota constitutes a vast ecosystem with a crucial role in establishing the mucosal immune system, and the intestinal microbiota of healthy adults is considered to be interindividually variable and intra-individually stable over long time periods (Eckburg et al., 2005; Jakobsson et al., 2010; Arumugam et al., 2011; Jalanka-Tuovinen et al., 2011). By contrast, the intestinal microbiota of infants is different from that of adults, with intestinal microbiota succession being affected by breast or formula feeding, weaning, diet, and unexpected life events, including infection and antibiotic treatment (Stark and Lee, 1982; Palmer et al., 2007; De Filippo et al., 2010; Koenig et al., 2011; Morotomi et al., 2011). The pathogenesis of KD has been suggested to involve a hyperimmune reaction in children who are genetically susceptible to variations in the normal flora; these variations are induced by environmental factors (Lee et al., 2007).

The intestinal microbiota of $\mathrm{KD}$ patients is characterized by a lack of Lactobacilli during the acute phase (Takeshita et al., 2002a) and the presence of HSP60-producing Gram-negative microbes (genera Acinetobacter, Enterobacter, Neisseria, and Veillonella) and Gram-positive cocci (genera Streptococcus and Staphylococcus) with the ability to induce V $\beta 2$ T cell expansion (Nagata et al., 2009). However, these studies on the intestinal profiles of $\mathrm{KD}$ patients were performed using culture-based methods.

Metagenomic analyses can reveal both the bacterial and viral compositions of the intestinal microbiota; thus, metagenomics can be used to identify potential pathogens in infectious diseases of unknown etiology (Kuroda et al., 2012). For instance, a metagenomic approach has revealed the presence of Streptococcus spp. in lymph node specimens of a KD patient, highlighting the possible role of these bacteria in KD (Katano et al., 2012).

In this study, a comparative metagenomic approach was used to characterize the differential microbiota compositions of $\mathrm{KD}$ patients by studying individual clinical specimens in a longitudinal manner. No study to date has performed longitudinal analysis of the microbial microbiota compositions of KD patients using a metagenomic approach. Indeed, although previous studies have suggested a possible role of the intestinal microbiota in the pathogenesis of $\mathrm{KD}$, they have relied only on culture-based methods for microbial detection (Takeshita et al., 2002a; Nagata et al., 2009). We therefore performed metagenomic analysis using a non-culture-based method to expand upon these results.

\section{Materials and Methods}

\section{Clinical Specimens Used for Comparative Metagenomic Analysis}

For the KD patient group, fecal samples were obtained at the time of admission (the acute phase), at the time of discharge 
(the convalescent phase), and at 4-6 months after the onset of $\mathrm{KD}$ (the non-acute phase). The study protocol was approved by the institutional medical ethics committee of the University of Tokyo, Tokyo Women's Medical University and the National Institute of Infectious Diseases in Japan (Approval No. 295), and it was conducted according to the Declaration of Helsinki Principles. Written informed consent was obtained from the parents of all children for publication of their individual details and accompanying images in this manuscript. The consent form is held by the authors' institution and is available for review.

\section{DNA Extraction from Fecal Samples}

Total DNA extraction was performed using a QIAamp ${ }^{\circledR}$ DNA Stool Mini Kit (QIAGEN, Tokyo, Japan) according to the manufacturer's instructions. To increase the recovery of bacterial DNA, particularly from Gram-positive bacteria, pretreatment with lytic enzymes was performed prior to extraction using the stool kit. Briefly, $100 \mathrm{mg}$ of fecal sample was suspended in $10 \mathrm{~mL}$ of Tris-EDTA buffer ( $\mathrm{pH} 7.5$ ), and $50 \mu \mathrm{L}$ of $100 \mathrm{mg} / \mathrm{mL}$ lysozyme type VI purified from chicken egg white (MPBIO, Derby, UK) and $50 \mu \mathrm{L}$ of $1 \mathrm{mg} / \mathrm{mL}$ purified achromopeptidase (Wako, Osaka, Japan) were added. The solution was incubated at $37^{\circ} \mathrm{C}$ for $1 \mathrm{~h}$ with mixing, $0.12 \mathrm{~g}$ of sodium dodecyl sulfate (final conc. $1 \%$ ) was added, and the suspension was mixed until it became clear. Next, $100 \mu \mathrm{L}$ of $20 \mathrm{mg} / \mathrm{mL}$ proteinase $\mathrm{K}$ (Wako) was added, followed by incubation at $55^{\circ} \mathrm{C}$ for $1 \mathrm{~h}$ with mixing. The cell lysate was then subjected to ethanol precipitation. The precipitant was dissolved in $1.6 \mathrm{~mL}$ of ASL buffer from the stool kit and subsequently purified using a QIAamp ${ }^{\circledR}$ DNA Stool Mini Kit (QIAGEN).

\section{DNA Library Preparation for Metagenomic Analysis and Short-read DNA Sequencing}

A DNA library was prepared using a Nextera ${ }^{\mathrm{TM}}$ DNA Sample Prep Kit (Illumina-compatible, EPICENTRE Biotechnologies, Madison, WI, USA), and DNA clusters were generated on a slide using a Cluster Generation Kit (version 2) with an Illumina cluster station (Illumina, San Diego, CA, USA) according to the manufacturer's instructions. The general procedure described in the standard protocol (Illumina) was performed to obtain standard $\sim 1.0 \times 10^{7}$ short reads for 1 lane. All of the sequencing runs for generating 126-mers were performed with a Genome Analyzer IIx using an Illumina Sequencing Kit (http://www.illumina.com/ systems/retired_gaiix/gaiix-kits.html). Fluorescence images were analyzed using Illumina base-calling pipeline (version 1.4.0) to obtain FASTQ-formatted sequence data. The short-read sequences have been deposited in DNA Data Bank of Japan (DDBJ; accession numbers: DRA000895 and DRA001171). All of the obtained DNA sequencing reads were aligned to a reference human genomic sequence using BWA-SW readmapping software (Li and Durbin, 2010), with quality trimming to remove low-quality reads. The remaining sequence reads were subjected to a megaBLAST search against a nucleotide database. The results of this search were analyzed and visualized using MEGAN version 4.62.3 (Huson et al., 2011), with a minimum support of 1 hit and a minimum score of 150 .

\section{Principal Component Analysis (PCA) and PERMANOVA Analysis}

The sequenced reads were assigned to a taxonomic hierarchy using MEGAN software following a megaBLAST homology search. The raw read counts were normalized by the total number of reads, and then PCA was performed using the R "prcomp" and "plot" functions. Permutational multivariate analysis of variance (PERMANOVA) with “ADONIS" was performed using 10,000 $\times$ permutations and the "bray" method with R's vegan package (Anderson, 2001).

\section{Linear Discriminant Analysis (LDA) Coupled With Effect Size Measurements (LEfSe)}

A metagenomic biomarker discovery approach, LEfSe, was used to identify the microbial components whose sequences were more abundant in the fecal samples of the KD patients during the acute phase than in those of the KD patients during the non-acute phase and the controls. For LEfSe, Kruskal-Wallis and pairwise Wilcoxon tests are performed, followed by LDA to assess the effect size of each differentially abundant taxon (Segata et al., 2011). In this study, a $p$-value of $<0.05$ was considered significant for both statistical methods. Bacteria with markedly increased numbers were defined as those with an LDA score $\left(\log _{10}\right)$ of over 2. Less than $0.01 \%$ of the total bacterial reads, corresponding with $\leq 10^{7} \mathrm{CFU} / \mathrm{g}$ feces, were omitted from further analysis because of low and unreliable read counts, although significant LDA scores were observed in LEfSe.

\section{Isolation of Streptococcus spp. and Species Determination Based on 16S-rRNA Gene}

Cultivation of Streptococcus spp. was performed using phenylethyl alcohol agar with $5 \%$ sheep blood or chocolate agar under anaerobic conditions at $37^{\circ} \mathrm{C}$ for $48 \mathrm{~h}$. The bacterial species present were determined by performing 16S-rRNA gene sequencing using the bacterial forward primer Bac27F (5'-AGAGTTTGGATCMTGGCTCAG-3') and the universal reverse primer Univ1492R (5'-CGGTTACCTTGTTACGACTT$3^{\prime}$ ) (Eden et al., 1991). The obtained sequences were searched against SILVA ribosomal RNA gene database to identify the bacterial species (Quast et al., 2013).

\section{Whole-Genome and Phylogenetic Analyses of Identified Streptococcus spp.}

A draft genome sequence was obtained by whole-genome sequencing using MiSeq with a NEXTERA XT library preparation kit (Illumina), followed by de novo assembly with A5-MiSeq pipeline (Tritt et al., 2012). The resulting scaffolds were annotated using RAST server (Aziz et al., 2008). Maximum likelihood phylogenetic analysis of Streptococcus 16S-rDNA was performed using MEGA 6.0 with 1000 bootstrap iterations (Tamura et al., 2013).

\section{Minimum Inhibitory Concentration (MIC) Testing}

MIC testing was performed using an Etest (bioMerieux, France) on Muller-Hinton agar (Difco, Augsburg), according to CLSI guidelines (CLSI, 2013). 


\section{Results}

\section{KD Patients Included in Comparative Metagenomic Analysis}

This study evaluated $28 \mathrm{KD}$ patients (15 males and 13 females, aged 1-114 mo; median of $25 \mathrm{mo}$ ). All of these patients were enrolled within 4 days of the onset of illness, with day 1 defined as the first day of fever, and they all met the diagnostic criteria for KD established by the American Heart Association (Newburger et al., 2004). All of the KD patients in the study received intravenous gamma globulin $(2 \mathrm{~g} / \mathrm{kg})$ and aspirin $(30-50 \mathrm{mg} / \mathrm{kg} /$ day). One male patient (patient P2) had a persistent fever despite receiving these therapies and was administered additional intravenous gamma globulin $(1 \mathrm{~g} / \mathrm{kg})$ and prednisolone sodium succinate $(2 \mathrm{mg} / \mathrm{kg} /$ day). This patient had transient dilatation of the coronary artery, whereas the other 27 patients showed no evidence of cardiac abnormalities.

In this study, the time of admission was defined as the acute phase, while 4-6 months after the onset of KD was considered the non-acute phase. The profiles of the participants, including the age, sex, concomitant symptoms and empirical antimicrobial treatment received, are shown in Table 1.

\section{Gut Microbiota Analysis Comparing the Acute and Non-acute Phases in KD Patients}

A total of 56 samples (28 samples each for the acute and non-acute phases) were collected, including two samples from each KD patient (Figure 1 and Table 1). Extracted DNA was subjected to metagenomic sequencing using an Illumina GAIIx next-generation DNA sequencer, and more than 10 million short 126-mer reads were obtained for each specimen. The short reads were classified at the family level of bacteria, with a threshold megaBLAST homology score of $=150$. Principal component analysis (PCA) was performed to elucidate the variations between the acute and non-acute phases of $\mathrm{KD}$. The results suggested that the gut microbiota was more variable during the acute phase than during the non-acute phase based on familylevel taxonomy (Figure 2A). PERMANOVA with $10,000 \times$ permutations revealed significant dissimilarity of the bacterial communities at the family level between the acute and non-acute

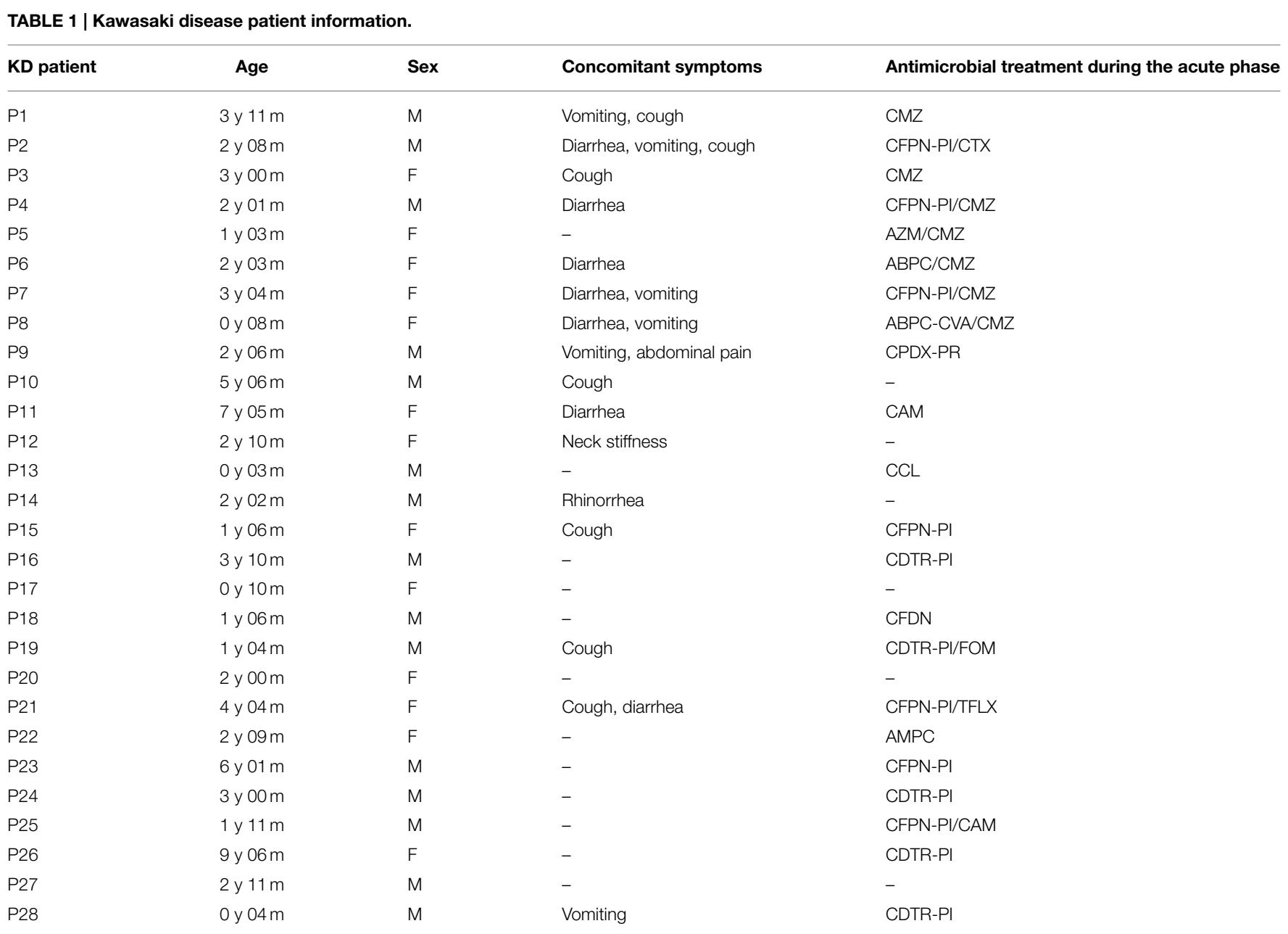

y, year; $m$, month. $M$, male; $F$, female. ABPC, ampicillin; ABPC-CVA, ampicillin-clavulanic acid; AMPC, amoxicillin; AZM, azithromycin; CAM, clarithromycin; CCL, cefaclor; CDTR-PI, cefditoren, pivoxil; CFDN, cefdinir; CFPN-PI, cefcapene pivoxil; CMZ, cefmetazole; CPDX-PR, cefpodoxime proxetil; CTX, cefotaxime; FOM, fosfomycin; TFLX, tosufloxacin. 


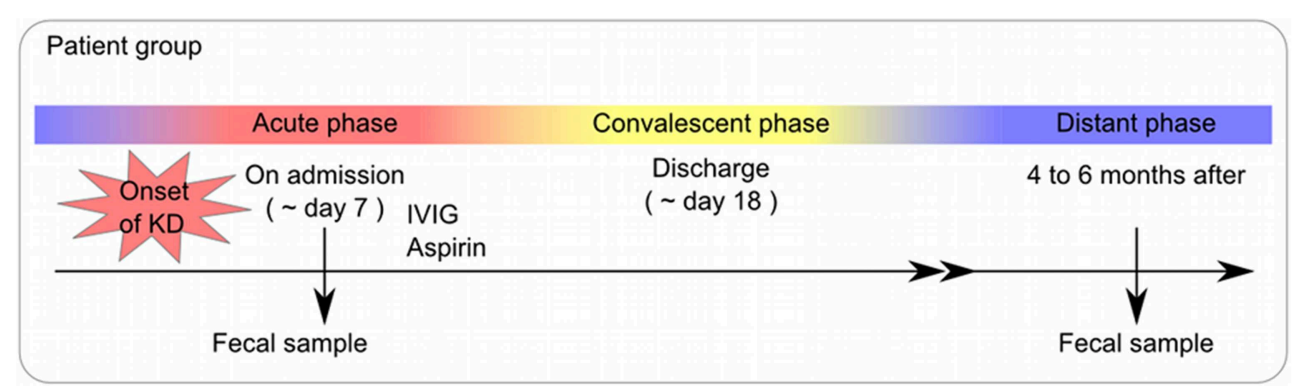

FIGURE 1 | The protocol for collection of clinical specimens.
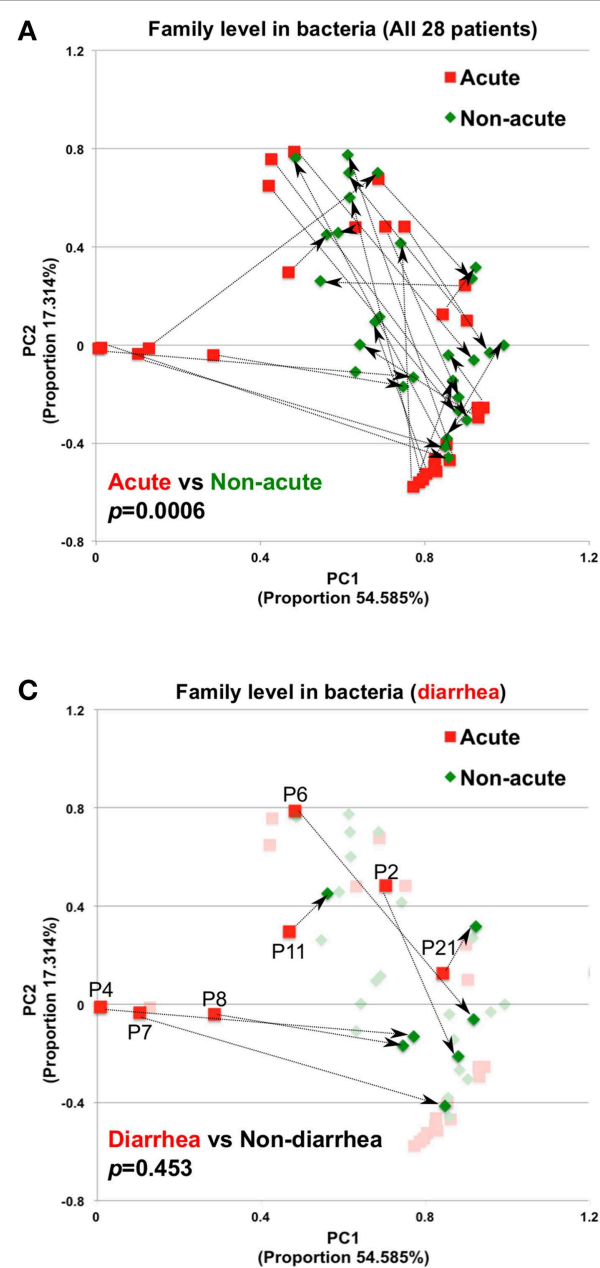

FIGURE 2 | Principal component analysis of gut microbiota compositions during the acute and non-acute phases of KD. (A) Relative abundance was estimated at the family taxonomic level (megaBLAST homology score threshold: $\geq 150$ ). The arrows indicate the corresponding pair for each patient for the acute and non-acute phases $(n=28)$. Some PCA plot
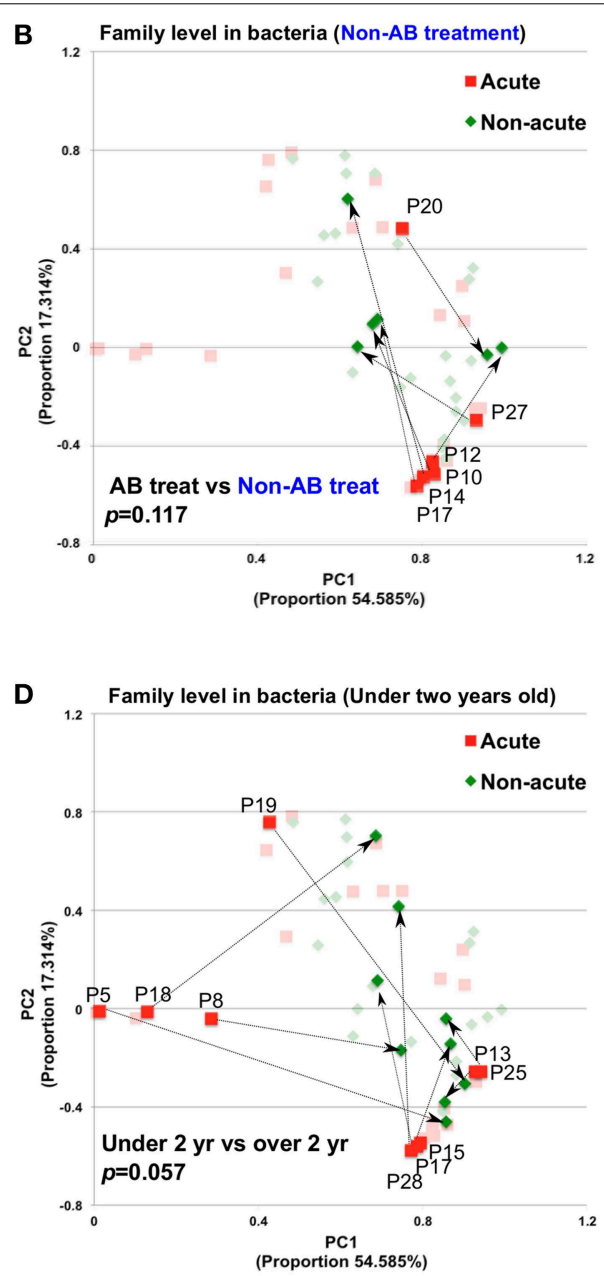

components were highlighted based on the indicated patient-related information, group of non-antimicrobial treatment (B), diarrhea symptom (C), and under two years old (D). To investigate the significance between the tested groups, PERMANOVA with "ADONIS" was performed using 10,000 × permutations, in addition to the "bray" method, with R's vegan package. phases of $\mathrm{KD}$ ( $F$-test $=3.7307, p=0.0006$ ) (Figure 2A). The components were highlighted based on the antimicrobial treatment, occurrence of diarrhea, and age group, indicating that such variations during the acute phase might be associated with patient-related factors. Further, the no antimicrobial treatment group during the acute phase had was clustered in the lower right area of the PCA plot (Figure 2B), whereas the diarrheapositive group during the acute phase exhibited a relatively 
scattered cluster in the PCA plot (Figure 2C); however, both groups during the non-acute phase were clustered together at the relative center of the plot (Figures $2 \mathrm{~B}, \mathrm{C}$ ). The patients in the antimicrobial treatment group did not always exhibit diarrhea symptoms (8 diarrhea/22 antimicrobial treatment), and PERMANOVA indicated no significant differences between the two subject groups, suggesting that the gut microbiota was not affected during the acute phase of $\mathrm{KD}$, regardless of the presence of antimicrobial treatment or diarrhea. The age factor showed a possible association with gut microbiota composition because the $p$-value was close to 0.05 when comparing subjects who were less than 2 years of age with those who were over 2 years of age (Figure 2D).

To determine the variations in gut microbiota composition between the acute and non-acute phases, linear discriminant analysis (LDA) coupled with effect size measurements (LEfSe) was applied to determine which taxa were enriched in the different groups according to metagenomic analysis (see detailed parameters in Materials and Methods). LEfSe determines which features (organisms, clades, operational taxonomic units, genes, or functions) are most likely to explain differences between classes by coupling standard tests for statistical significance (between the acute and non-acute phases in this study) with additional tests of biological consistency and effect relevance (Segata et al., 2011). The obtained metagenomic reads were classified at the genus level (Figure 3). Although LEfSe revealed that the genera Rothia and Staphylococcus were the most abundant during the acute phase, this dominance was not observed in all of the patients (Figure 3). Regardless, relatively increased numbers of Ruminococcus, Blautia, Faecalibacterium, and Roseburia bacteria were observed during the non-acute phase of KD (Figure 3), indicating that these genera was possibly related to remission in $\mathrm{KD}$ patients.

The above genus classifications did not reveal which common features were most likely to explain the differences between the acute and non-acute phases in the tested $\mathrm{KD}$ patients $(n=28)$. Because both pathogenic and non-pathogenic species may be included within one bacterial genus, we speculated that genus classifications would not reveal certain potential pathogens involved in KD pathogenesis; thus, further taxonomic

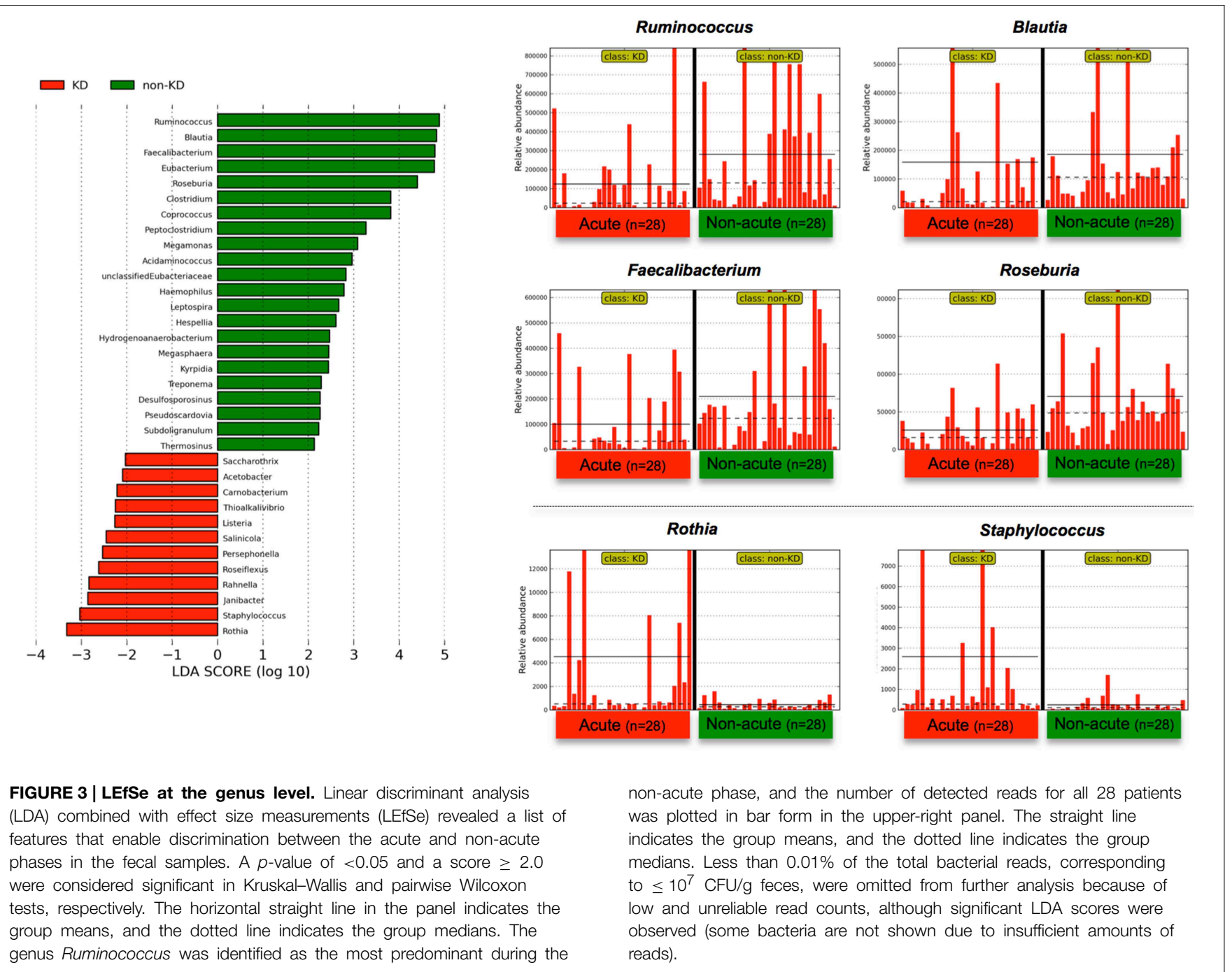


classifications at the species level may allow for effective detection of KD-related pathogens (Figure 4). Roseburia spp. were relatively abundant during the non-acute phase and could be identified at the genus level (Figure 3), whereas Streptococcus spp. were predominantly identified during the acute phase, suggesting that some Streptococcus spp., including S. pneumoniae, $S$. oralis and other strains, are candidate KDrelated pathogens (Figure 4). Staphylococcus hyicus might have been misidentified due to an insufficient amount of reads (less than $0.01 \%$ of the population; $<1000$ of the assigned reads), although the LDA score was significant.

Indeed, Streptococcus spp. were highly abundant in the gut microbiotas of some of the KD patients; for example, $77 \%$ of the bacterial reads of one KD patient (the acute phase in P7) were from Streptococcus. However, the above-mentioned $S$. pneumoniae and oralis species were not cultured from feces during the acute phase of $\mathrm{KD}$ under conventional aerobic cultivation on a phenylethyl alcohol agar plate with 5\% sheep blood in screening for Streptococcus spp., while anaerobic cultivation on chocolate agar resulted in positive Streptococcus colonies. In fact, fifty colonies of Streptococcus spp. were isolated from P7-feces on chocolate agar under anaerobic conditions with incubation at $37^{\circ} \mathrm{C}$ for $16 \mathrm{~h}$, and then $16 \mathrm{~S}$-rDNA sequencing was performed to determine the bacterial species present. The results suggested that seven Streptococcus spp. were unique isolates (P7-Anaero4, P7-Anaero13, P7-Anaero24, P7-Anaero25, P7Anaero36, P7-Anaero39, and P7-Anaero45) (Figure 5C). Using the draft genome sequences of seven P7-Streptococcus isolates, including publicly available Streptococcus spp. complete genomes (23 species), the metagenomic short reads of all 56 fecal samples were classified at the species level by a megaBLAST search and LEfSe. The results suggested that the amounts of the six P7-fecesrelated Streptococcus isolates (P7-Anaero4, 13, 24, 36, 39, and 45, but not P7-Anaero25) and the five detected Streptococcus spp. (S. pneumonia, pseudopneumoniae, oralis, gordonii, and sanguinis) were apparently increased during the acute phase in most of the KD patients, including $\mathrm{P} 7$, whereas $S$. pasteurianus was increased during the non-acute phase (Figures 5A,B). Intriguingly, the top 4 most abundant positive isolates were P7-feces-related Streptococcus spp. (P7-Anaero4, 45, 24, and 36) rather than defined pathogenic Streptococcus species, and all positively detected Streptococcus spp. were classified within a taxonomic lineage closely related to $S$. oralis or pneumonia (Figure 5C). All P7-feces-related Streptococcus isolates showed susceptibility to most antimicrobial agents, including cephem, indicating that the detection of abundant P7-feces-related isolates was most likely not correlated with antimicrobial selection.

Based on species-level identification, the first and second highest hits with identical BLAST scores constituted $17.9 \%$ of all of the short reads, which were mostly homologous to rRNA genes; thus, $82.1 \%$ of the short reads could be assigned to a unique top hit. Although the obtained 126-mer short reads might not have been sufficiently long for correct species assignments, the BLAST search results suggested that the above-mentioned P7-related Streptococcus groups were highly abundant during the acute phase of $\mathrm{KD}$, in contrast with other pathogenic Streptococcus spp., such as S. pyogenes, dysgalactiae, mutans, and pasteurianus. The significant detection of unique isolates in $\mathrm{KD}$ patients implies a possible association of $\mathrm{KD}$ with uncharacterized Streptococcus spp.

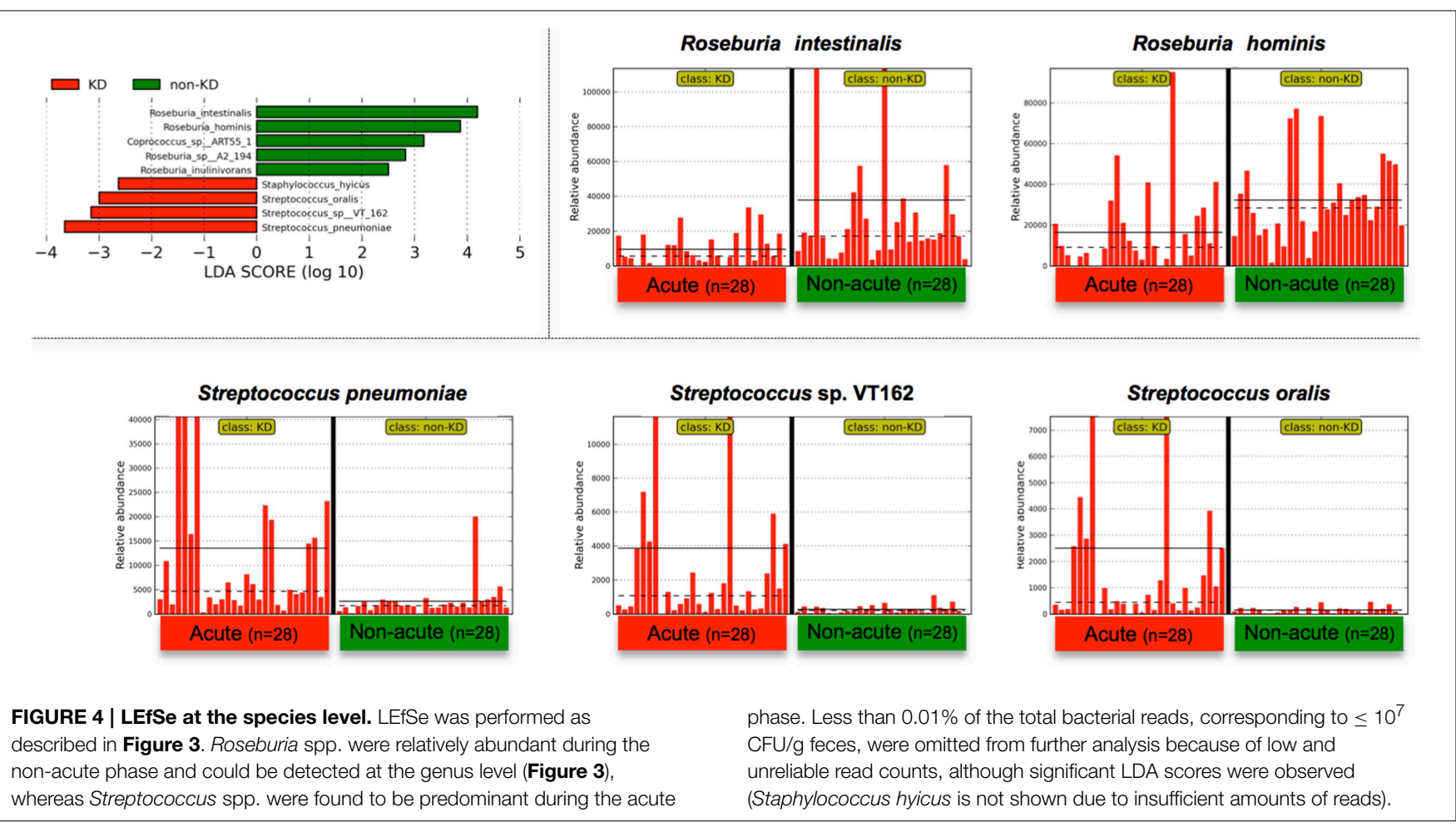




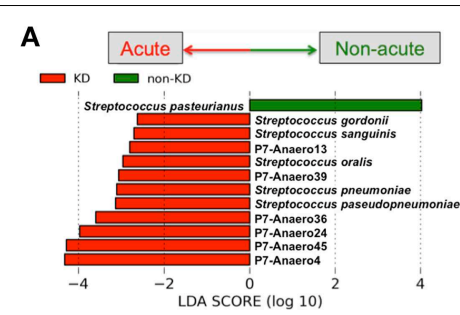

C

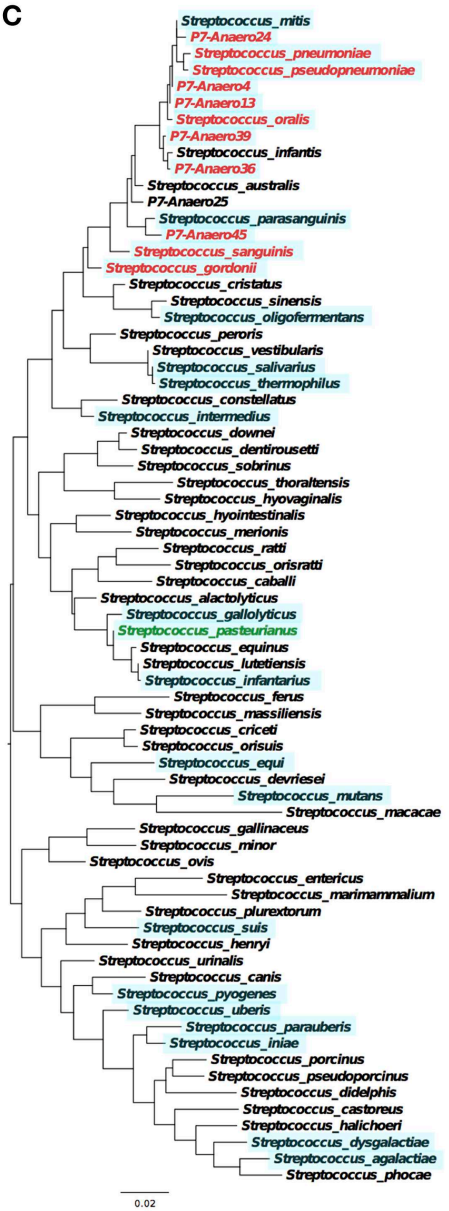

B
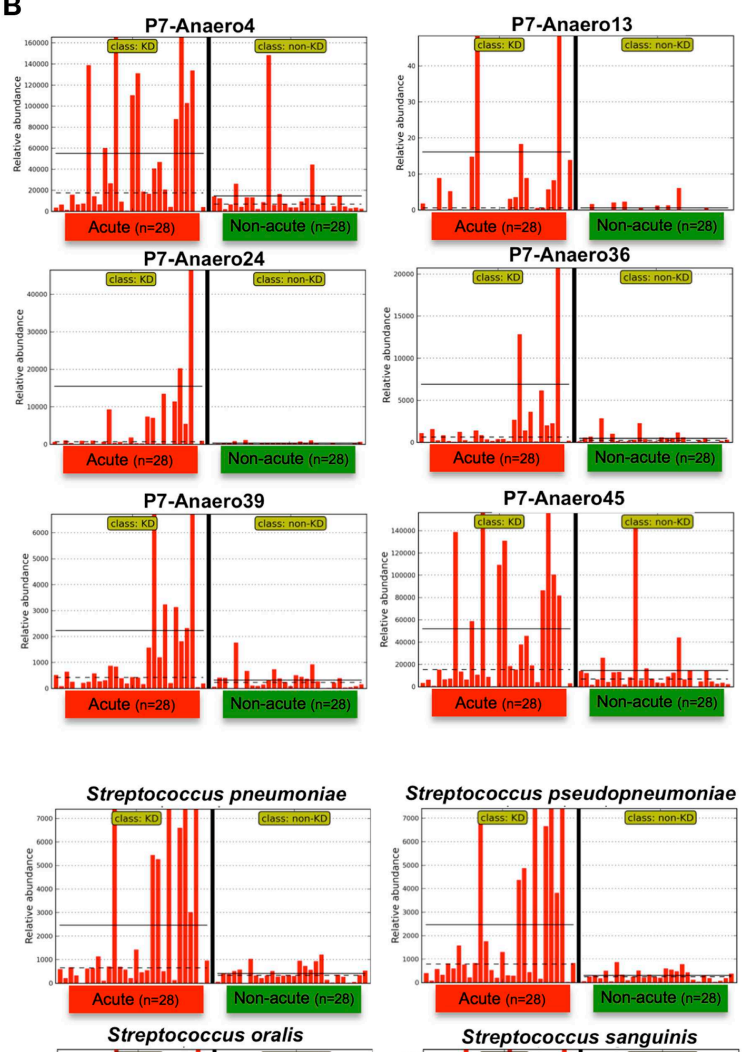

Streptococcus pseudopneumoniae
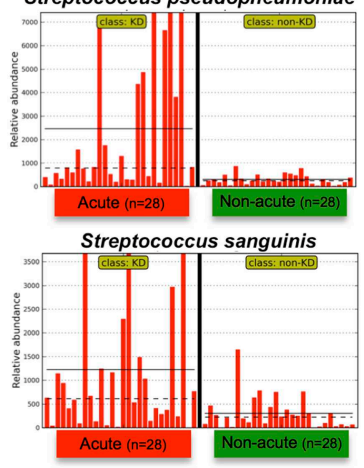

Streptococcus pasteurianus

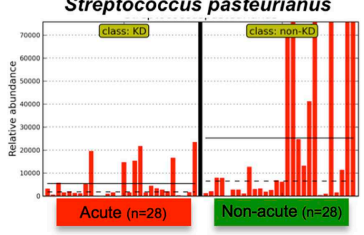

FIGURE 5 | LEfSe of P7-related Streptococcus isolates performed using Streptococcus genome database. (A) The type of Streptococcus spp. markedly differed between the acute and non-acute phases of KD. (B) The relative abundance of detected reads for the patients $(n=28)$ was plotted for each Streptococcus spp. The horizontal straight line indicates the group means, and the dotted line indicates the group medians in the panel. (C) Maximum likelihood phylogenetic analysis of Streptococcus 16S-rDNA. The Streptococcus species in red and green are those with increased abundance during the acute and non-acute phases of $\mathrm{KD}$, respectively. The complete and draft genome sequences used for the megaBLAST search are highlighted with a light blue background.

\section{Discussion}

Various bacterial and viral agents have been reported to be associated with KD pathogenesis (Johnson and Azimi, 1985; Catalano-Pons et al., 2005; Wang et al., 2005; Joshi et al., 2011), but these speculations have been controversial (Wang et al., 2005). Colonization by normal microbiota variants have been suggested to induce a dysregulation in the immune systems of children with a pre-existing genetic defect in immune maturation, leading to a hyperimmune reaction and the development of KD (Lee et al., 2007). In this study, the possible pathogens detected in the KD patients varied for each individual patient; thus, every identified pathogen represented a potential candidate. Regarding virus species, human adenovirus (HAdV) species F was detected in one out of twenty-eight of the patients, despite the absence of gastrointestinal manifestations in that patient. Thus, HAdV was not commonly detected, and no sequences from either other viruses or previously reported pathogens were detected in any of the other KD samples. 
Although the gut microbiota markedly differed at the genus level between the acute and non-acute phases of KD (Figure 3), we speculated that classification at the species level might be appropriate for identifying disease-associated bacteria because a genus includes species that have varying effects on human health [e.g., S. pyogenes infections include acute rheumatic fever, pharyngitis, impetigo and streptococcal toxic shock syndrome (STSS); S. pneumoniae causes many types of pneumococcal infections other than pneumonia; and $S$. mutans is a significant contributor to tooth decay in the human oral cavity].

The findings of this study suggested that notable Streptococcus spp. in the mitis group, including S. pneumonia, pseudopneumoniae, mitis, oralis, gordonii, and sanguinis, were highly abundant in the fecal samples during the acute phase (Figures 4, 5); therefore, members of the mitis group of Streptococci could be present in the bacterial flora of $\mathrm{KD}$ patients. The mitis group comprises agents that contribute to oral biofilms, dental plaques, and infective endocarditis, disease processes that involve bacteria-bacteria and bacteria-host interactions (Whatmore et al., 2000). To further elucidate the association between Streptococcus spp. and KD in this study, we isolated a unique Streptococcus spp. (Figure 5C) and then performed whole-genome sequencing and a megaBLAST homology search. The results revealed a significant abundance of KD-derived Streptococcus isolates during the acute phase of the disease (Figure 5). Intriguingly, a recent paper has reported metagenomic analysis of the human gut microbiome in liver cirrhosis patients, suggesting that oral commensals, including Streptococcus spp., invade the gut in patients with liver cirrhosis (Qin et al., 2014) and implying that uncharacterized Streptococcus spp. could be potential biomarkers/pathogens for diseases with unknown etiologies.

A SAg hypothesis for $\mathrm{KD}$ on the etiology remains inconclusive, the involvement of single or multiple SAgs on $\mathrm{T}$-cell $\mathrm{V} \beta$ repertoires has been speculated for the $\mathrm{KD}$ pathogenesis (Matsubara and Fukaya, 2007). A number of studies have found primarily V $\beta 2$ expansion (Abe et al., 1992; Leung et al., 1995; Yoshioka et al., 1999) linking to the V $\beta 2$ specific SAg such as toxic shock syndrome toxin-1 (TSST-1) and SpeC (Nur-Ur Rahman et al., 2011), although there is no direct evidence to suggest SAg involvement. STSS is significantly more frequent in group A B-hemolytic streptococcal (GAS) patients than in groups $B, C$, and $G$ streptococcal patients. GAS produces a multitude of surface-bound and secreted virulence factors causing resistance to phagocytosis, complement deposition, antibody opsonization, and neutrophil killing mechanisms, leading to overactive immune response and subverting host innate immune defenses (Walker et al., 2014). Although the isolation of SAg-positive Streptococcus from KD patients has been reported (Nagata et al., 2009; Leahy et al., 2012), group A B-hemolytic streptococcal (GAS) might not contribute to the pathogenesis of this disease because a rapid antigen detection test (RADT) and proper antibiotic treatment prevents GAS pharyngitis during the initial episodes of acute rheumatic fever (Gerber et al., 2009). Because the KD patients in this study (Table 1) were empirically treated with antibiotics during the early stages of the disease, the results may reflect the effects of the antibiotic therapy. To address this issue, a full comparison of $\mathrm{KD}$ patients who have and have not received antibiotic therapy should be performed in a large, controlled study. It is also possible that antibiotic therapy has an adverse effect on the pathogenesis of KD. Further investigation of the role of Streptococcus spp. in the pathogenesis of KD is therefore merited.

Our previous metagenomic approach indicated that Streptococcus spp. were present in the lymph node specimen of one KD patient, highlighting the possible role of these bacteria in KD (Katano et al., 2012). To identify the SAg homologs, all short reads and coding sequences of the 7 isolates (P7-Anaero4, 13, $24,25,36,39$, and 45 ) were subjected to a PSI-BLAST homology search against "superantigen, staphylococcal/streptococcal toxin, bacterial (IPR013307)" orthologous proteins; however, no significant match has been found thus far (data not shown). In addition, some sequences from each sample were classified as "Not assigned" in metagenomic analysis, and new pathogenic agents remain to be characterized for some of these unidentified sequences.

The gut microbiota in the non-acute phase of KD (the distant phase) was similar in each patient, and the genera Ruminococcus, Roseburia and Faecalibacterium were predominant (Figure 3). In previous reports, an observed elevation in LPS-binding neutrophils or plasma proteins has been observed, suggesting that LPS infusion followed by disruption in intestinal mucosal barrier function might be involved in the pathogenesis of this disease (Takeshita et al., 1999, 2002b); therefore, a well-balanced commensal gut microbiota contributes to the mucosal barrier function of the intestine. Prebiotics, probiotics, and combination synbiotics modulate the balance of the intestinal microbiota and may help to prevent the onset of $\mathrm{KD}$ to improve patient prognosis (Bosscher et al., 2009).

The microbiota of KD patients was comprehensively analyzed in this study. Our findings suggest that markedly increased amounts of Streptococcus spp. are present in the gut microbiotas of acute-phase KD patients and that this difference in microbiota composition might be related to $\mathrm{KD}$ pathogenesis.

\section{Author Contributions}

$\mathrm{AK}$ and $\mathrm{HH}$ collected clinical specimens from the KD patients. TS, KK, and AY performed the metagenomic sequencing and statistical and bioinformatics analyses. AK and MK participated in the design of the study, performed statistical analysis, and drafted the manuscript. All authors read and approved the final manuscript.

\section{Acknowledgments}

This work was supported by grants from the NPO Japan Kawasaki Disease Research Center in 2010/2011 and from the Ministry of Education, Science, Sports and Culture, Grant-in-Aid for Scientific Research (C) in 2011 (23590527)/2014 (26460542). The funders had no role in the study design, data collection and analysis, decision to publish, or manuscript preparation. 


\section{References}

Abe, J., Kotzin, B. L., Jujo, K., Melish, M. E., Glode, M. P., Kohsaka, T., et al. (1992). Selective expansion of $\mathrm{T}$ cells expressing $\mathrm{T}$-cell receptor variable regions $\mathrm{V}$ beta 2 and $\mathrm{V}$ beta 8 in Kawasaki disease. Proc. Natl. Acad. Sci. U.S.A. 89, 4066-4070. doi: 10.1073/pnas.89.9.4066

Anderson, M. J. (2001). A new method for non-parametric multivariate analysis of variance. Aust. Ecol. 26, 32-46. doi: 10.1111/j.1442-9993.2001.01070.pp.x

Arumugam, M., Raes, J., Pelletier, E., Le Paslier, D., Yamada, T., Mende, D. R., et al. (2011). Enterotypes of the human gut microbiome. Nature 473, 174-180. doi: 10.1038/nature09944

Aziz, R. K., Bartels, D., Best, A. A., Dejongh, M., Disz, T., Edwards, R. A., et al. (2008). The RAST Server: rapid annotations using subsystems technology. BMC Genomics 9:75. doi: 10.1186/1471-2164-9-75

Bosscher, D., Breynaert, A., Pieters, L., and Hermans, N. (2009). Food-based strategies to modulate the composition of the intestinal microbiota and their associated health effects. J. Physiol. Pharmacol. 60(Suppl. 6), 5-11.

Catalano-Pons, C., Quartier, P., Leruez-Ville, M., Kaguelidou, F., Gendrel, D., Lenoir, G., et al. (2005). Primary cytomegalovirus infection, atypical Kawasaki disease, and coronary aneurysms in 2 infants. Clin. Infect. Dis. 41, e53-56. doi: $10.1086 / 432578$

CLSI. (2013). Clinical and Laboratory Standards Institute. 2013. Performance Standards for Antimicrobial Susceptibility Testing; Twenty-third Informational Supplement. CLSI Document M100-S23. Wayne, PA: Clinical and Laboratory Standards Institute.

De Filippo, C., Cavalieri, D., Di Paola, M., Ramazzotti, M., Poullet, J. B., Massart, S., et al. (2010). Impact of diet in shaping gut microbiota revealed by a comparative study in children from Europe and rural Africa. Proc. Natl. Acad. Sci. U.S.A. 107, 14691-14696. doi: 10.1073/pnas.1005963107

Duong, T. T., Silverman, E. D., Bissessar, M. V., and Yeung, R. S. (2003). Superantigenic activity is responsible for induction of coronary arteritis in mice: an animal model of Kawasaki disease. Int. Immunol. 15, 79-89. doi: 10.1093/intimm/dxg007

Eckburg, P. B., Bik, E. M., Bernstein, C. N., Purdom, E., Dethlefsen, L., Sargent, M., et al. (2005). Diversity of the human intestinal microbial flora. Science 308, 1635-1638. doi: 10.1126/science.1110591

Eden, P. A., Schmidt, T. M., Blakemore, R. P., and Pace, N. R. (1991). Phylogenetic analysis of Aquaspirillum magnetotacticum using polymerase chain reactionamplified 16S rRNA-specific DNA. Int. J. Syst. Bacteriol. 41, 324-325. doi: 10.1099/00207713-41-2-324

Fujita, Y., Nakamura, Y., Sakata, K., Hara, N., Kobayashi, M., Nagai, M., et al. (1989). Kawasaki disease in families. Pediatrics 84, 666-669.

Gerber, M. A., Baltimore, R. S., Eaton, C. B., Gewitz, M., Rowley, A. H., Shulman, S. T., et al. (2009). Prevention of rheumatic fever and diagnosis and treatment of acute Streptococcal pharyngitis: a scientific statement from the American Heart Association Rheumatic Fever, Endocarditis, and Kawasaki Disease Committee of the Council on Cardiovascular Disease in the Young, the Interdisciplinary Council on Functional Genomics and Translational Biology, and the Interdisciplinary Council on Quality of Care and Outcomes Research: endorsed by the American Academy of Pediatrics. Circulation 119, 1541-1551. doi: 10.1161/CIRCULATIONAHA.109.191959

Holman, R. C., Christensen, K. Y., Belay, E. D., Steiner, C. A., Effler, P. V., Miyamura, J., et al. (2010). Racial/ethnic differences in the incidence of Kawasaki syndrome among children in Hawaii. Hawaii Med. J. 69, 194-197.

Huson, D. H., Mitra, S., Ruscheweyh, H. J., Weber, N., and Schuster, S. C. (2011). Integrative analysis of environmental sequences using MEGAN4. Genome Res. 21, 1552-1560. doi: 10.1101/gr.120618.111

Jakobsson, H. E., Jernberg, C., Andersson, A. F., Sjölund-Karlsson, M., Jansson, J. K., and Engstrand, L. (2010). Short-term antibiotic treatment has differing longterm impacts on the human throat and gut microbiome. PLoS ONE 5:e9836. doi: 10.1371 /journal.pone.0009836

Jalanka-Tuovinen, J., Salonen, A., Nikkilä, J., Immonen, O., Kekkonen, R., Lahti, L., et al. (2011). Intestinal microbiota in healthy adults: temporal analysis reveals individual and common core and relation to intestinal symptoms. PLoS ONE 6:e23035. doi: 10.1371/journal.pone.0023035

Johnson, D., and Azimi, P. (1985). Kawasaki disease associated with Klebsiella pneumoniae bacteremia and parainfluenza type 3 virus infection. Pediatr. Infect. Dis. 4, 100. doi: 10.1097/00006454-198501000-00024
Joshi, A. V., Jones, K. D., Buckley, A. M., Coren, M. E., and Kampmann, B. (2011). Kawasaki disease coincident with influenza A H1N1/09 infection. Pediatr. Int. 53, e1-e2. doi: 10.1111/j.1442-200X.2010.03280.x

Katano, H., Sato, S., Sekizuka, T., Kinumaki, A., Fukumoto, H., Sato, Y., et al. (2012). Pathogenic characterization of a cervical lymph node derived from a patient with Kawasaki disease. Int. J. Clin. Exp. Pathol. 5, 814-823.

Kawasaki, T. (1967). [Acute febrile mucocutaneous syndrome with lymphoid involvement with specific desquamation of the fingers and toes in children]. Arerugi 16, 178-222.

Koenig, J. E., Spor, A., Scalfone, N., Fricker, A. D., Stombaugh, J., Knight, R., et al. (2011). Succession of microbial consortia in the developing infant gut microbiome. Proc. Natl. Acad. Sci. U.S.A. 108(Suppl. 1), 4578-4585. doi: 10.1073/pnas.1000081107

Kuroda, M., Sekizuka, T., Shinya, F., Takeuchi, F., Kanno, T., Sata, T., et al. (2012). Detection of a possible bioterrorism agent, Francisella sp., in a clinical specimen by use of next-generation direct DNA sequencing. J. Clin. Microbiol. 50, 1810-1812. doi: 10.1128/JCM.06715-11

Leahy, T. R., Cohen, E., and Allen, U. D. (2012). Incomplete Kawasaki disease associated with complicated Streptococcus pyogenes pneumonia: a case report. Can J Infect Dis Med Microbiol 23, 137-139.

Lee, K. Y., Han, J. W., and Lee, J. S. (2007). Kawasaki disease may be a hyperimmune reaction of genetically susceptible children to variants of normal environmental flora. Med. Hypothes. 69, 642-651. doi: 10.1016/j.mehy.2006.12.051

Leung, D. Y., Meissner, C., Fulton, D., and Schlievert, P. M. (1995). The potential role of bacterial superantigens in the pathogenesis of Kawasaki syndrome. J. Clin. Immunol. 15, 11S-17S. doi: 10.1007/BF01540888

Li, H., and Durbin, R. (2010). Fast and accurate long-read alignment with Burrows-Wheeler transform. Bioinformatics 26, 589-595. doi: 10.1093/bioinformatics/btp698

Matsubara, K., and Fukaya, T. (2007). The role of superantigens of group A Streptococcus and Staphylococcus aureus in Kawasaki disease. Curr. Opin. Infect. Dis. 20, 298-303. doi: 10.1097/QCO.0b013e3280964d8c

Morotomi, N., Fukuda, K., Nakano, M., Ichihara, S., Oono, T., Yamazaki, T., et al. (2011). Evaluation of intestinal microbiotas of healthy Japanese adults and effect of antibiotics using the 16S ribosomal RNA gene based clone library method. Biol. Pharmaceut. Bull. 34, 1011-1020. doi: 10.1248/bpb.34.1011

Nagata, S., Yamashiro, Y., Ohtsuka, Y., Shimizu, T., Sakurai, Y., Misawa, S., et al. (2009). Heat shock proteins and superantigenic properties of bacteria from the gastrointestinal tract of patients with Kawasaki disease. Immunology 128, 511-520. doi: 10.1111/j.1365-2567.2009.03135.x

Nagi-Miura, N., Shingo, Y., Adachi, Y., Ishida-Okawara, A., Oharaseki, T., Takahashi, K., et al. (2004). Induction of coronary arteritis with administration of CAWS (Candida albicans water-soluble fraction) depending on mouse strains. Immunopharmacol. Immunotoxicol. 26, 527-543. doi: 10.1081/IPH200042295

Nakamura, T., Yamamura, J., Sato, H., Kakinuma, H., and Takahashi, H. (2007). Vasculitis induced by immunization with Bacillus Calmette-Guerin followed by atypical mycobacterium antigen: a new mouse model for Kawasaki disease. FEMS Immunol. Med. Microbiol. 49, 391-397. doi: 10.1111/j.1574695X.2007.00217.x

Nakamura, Y., Yashiro, M., Uehara, R., Oki, I., Watanabe, M., and Yanagawa, H. (2008). Monthly observation of the number of patients with Kawasaki disease and its incidence rates in Japan: chronological and geographical observation from nationwide surveys. J. Epidemiol. 18, 273-279. doi: 10.2188/jea.JE2008030

Nakamura, Y., Yashiro, M., Uehara, R., Sadakane, A., Tsuboi, S., Aoyama, Y., et al. (2012). Epidemiologic features of Kawasaki disease in Japan: results of the 20092010 nationwide survey. J. Epidemiol. 22, 216-221. doi: 10.2188/jea.JE20110126 Newburger, J. W., Takahashi, M., Beiser, A. S., Burns, J. C., Bastian, J., Chung, K. J., et al. (1991). A single intravenous infusion of gamma globulin as compared with four infusions in the treatment of acute Kawasaki syndrome. N. Eng. J. med. 324, 1633-1639. doi: 10.1056/NEJM199106063242305

Newburger, J. W., Takahashi, M., Gerber, M. A., Gewitz, M. H., Tani, L. Y., Burns, J. C., et al. (2004). Diagnosis, treatment, and long-term management of Kawasaki disease: a statement for health professionals from the Committee on Rheumatic Fever, Endocarditis, and Kawasaki Disease, Council on Cardiovascular Disease in the Young, American Heart Association. Pediatrics 114, 1708-1733. doi: 10.1542/peds.2004-2182 
Nur-Ur Rahman, A. K., Bonsor, D. A., Herfst, C. A., Pollard, F., Peirce, M., Wyatt, A. W., et al. (2011). The $\mathrm{T}$ cell receptor beta-chain second complementarity determining region loop (CDR2beta governs $\mathrm{T}$ cell activation and Vbeta specificity by bacterial superantigens. J. Biol. Chem. 286, 4871-4881. doi: 10.1074/jbc.M110.189068

Ohno, N. (2004). Murine model of Kawasaki disease induced by mannoproteinbeta-glucan complex, CAWS, obtained from Candida albicans. Jpn. J. Infect. Dis. 57, S9-S10.

Onouchi, Y. (2012). Genetics of Kawasaki disease: what we know and don't know. Circ. J. 76, 1581-1586. doi: 10.1253/circj.CJ-12-0568

Onouchi, Y., Ozaki, K., Burns, J. C., Shimizu, C., Terai, M., Hamada, H., et al. (2012). A genome-wide association study identifies three new risk loci for Kawasaki disease. Nat. Genet. 44, 517-521. doi: 10.1038/ ng. 2220

Palmer, C., Bik, E. M., Digiulio, D. B., Relman, D. A., and Brown, P. O. (2007). Development of the human infant intestinal microbiota. PLoS Biol. 5:e177. doi: 10.1371/journal.pbio.0050177

Principi, N., Rigante, D., and Esposito, S. (2013). The role of infection in Kawasaki syndrome. J. Infect. 67, 1-10. doi: 10.1016/j.jinf.2013.04.004

Qin, N., Yang, F., Li, A., Prifti, E., Chen, Y., Shao, L., et al. (2014). Alterations of the human gut microbiome in liver cirrhosis. Nature 513, 59-64. doi: 10.1038/nature 13568

Quast, C., Pruesse, E., Yilmaz, P., Gerken, J., Schweer, T., Yarza, P., et al. (2013). The SILVA ribosomal RNA gene database project: improved data processing and web-based tools. Nucleic Acids Res. 41, D590-D596. doi: 10.1093/nar/ gks1219

Segata, N., Izard, J., Waldron, L., Gevers, D., Miropolsky, L., Garrett, W. S., et al. (2011). Metagenomic biomarker discovery and explanation. Genome Biol. 12:R60. doi: 10.1186/gb-2011-12-6-r60

Stark, P. L., and Lee, A. (1982). The microbial ecology of the large bowel of breastfed and formula-fed infants during the first year of life. J. Med. Microbiol. 15, 189-203. doi: 10.1099/00222615-15-2-189

Takeshita, S., Kobayashi, I., Kawamura, Y., Tokutomi, T., and Sekine, I. (2002a). Characteristic profile of intestinal microflora in Kawasaki disease. Acta Paediatr. 91, 783-788. doi: 10.1111/j.1651-2227.2002.tb03327.x

Takeshita, S., Nakatani, K., Kawase, H., Seki, S., Yamamoto, M., Sekine, I., et al. (1999). The role of bacterial lipopolysaccharide-bound neutrophils in the pathogenesis of Kawasaki disease. J. Infect. Dis. 179, 508-512. doi: $10.1086 / 314600$

Takeshita, S., Tsujimoto, H., Kawase, H., Kawamura, Y., and Sekine, I. (2002b). Increased levels of lipopolysaccharide binding protein in plasma in children with kawasaki disease. Clin. Diagn. Lab. Immunol. 9, 205-206. doi: 10.1128/cdli.9.1.205-206.2002
Tamura, K., Stecher, G., Peterson, D., Filipski, A., and Kumar, S. (2013). MEGA6: Molecular Evolutionary Genetics Analysis version 6.0. Mol. Biol. Evol. 30, 2725-2729. doi: 10.1093/molbev/mst197

Tritt, A., Eisen, J. A., Facciotti, M. T., and Darling, A. E. (2012). An integrated pipeline for de novo assembly of microbial genomes. PLOS ONE 7:e42304. doi: 10.1371/journal.pone.0042304

Uehara, R., and Belay, E. D. (2012). Epidemiology of Kawasaki disease in Asia, Europe, and the United States. J. Epidemiol. 22, 79-85. doi: 10.2188/jea.JE20110131

Walker, M. J., Barnett, T. C., Mcarthur, J. D., Cole, J. N., Gillen, C. M., Henningham, A., et al. (2014). Disease manifestations and pathogenic mechanisms of group a Streptococcus. Clin. Microbiol. Rev. 27, 264-301. doi: 10.1128/CMR.00101-13

Wang, C. L., Wu, Y. T., Liu, C. A., Kuo, H. C., and Yang, K. D. (2005). Kawasaki disease: infection, immunity and genetics. Pediatr. Infect. Dis. J. 24, 998-1004. doi: 10.1097/01.inf.0000183786.70519.fa

Whatmore, A. M., Efstratiou, A., Pickerill, A. P., Broughton, K., Woodard, G., Sturgeon, D., et al. (2000). Genetic relationships between clinical isolates of Streptococcus pneumoniae, Streptococcus oralis, and Streptococcus mitis: characterization of "Atypical" pneumococci and organisms allied to S. mitis harboring S. pneumoniae virulence factor-encoding genes. Infect. Immun. 68, 1374-1382. doi: 10.1128/IAI.68.3.1374-1382.2000

Yokota, S., Tsubaki, K., Kuriyama, T., Shimizu, H., Ibe, M., Mitsuda, T., et al. (1993). Presence in Kawasaki disease of antibodies to mycobacterial heat-shock protein HSP65 and autoantibodies to epitopes of human HSP65 cognate antigen. Clin. Immunol. Immunopathol. 67, 163-170. doi: 10.1006/clin.1993.1060

Yoshioka, T., Matsutani, T., Iwagami, S., Toyosaki-Maeda, T., Yutsudo, T., Tsuruta, Y., et al. (1999). Polyclonal expansion of TCRBV2- and TCRBV6-bearing $\mathrm{T}$ cells in patients with Kawasaki disease. Immunology 96, 465-472. doi: 10.1046/j.1365-2567.1999.00695.x

Conflict of Interest Statement: The authors declare that the research was conducted in the absence of any commercial or financial relationships that could be construed as a potential conflict of interest.

Copyright (๑) 2015 Kinumaki, Sekizuka, Hamada, Kato, Yamashita and Kuroda. This is an open-access article distributed under the terms of the Creative Commons Attribution License (CC BY). The use, distribution or reproduction in other forums is permitted, provided the original author(s) or licensor are credited and that the original publication in this journal is cited, in accordance with accepted academic practice. No use, distribution or reproduction is permitted which does not comply with these terms. 